\title{
Evaluation and Comparative Study of Wireless Sensors Networks Hierarchical Protocols
}

\author{
Hassan Echoukairi, Khalid Bouragba and Mohammed Ouzzif \\ Laboratory of Computer Networks, Telecommunications and Multimedia CED Engineering Sciences Higher School of Technology \\ Hassan II University of Casablanca, Morocco
}

\begin{abstract}
In this paper, we analyze the performances of wireless sensor networks in hierarchical protocols LEACH, LEACH-C and PEGASIS in terms of energy dissipation, throughput, PDR (packet delivery ratio) as a function of the nodes density and base station location by using the NS2 simulator. Also, we discuss the effect of the varying node density for each position of a base station in the sensor filed on this metric parameters.
\end{abstract}

Key words: WSN (wireless sensor network), routing protocols, performance evaluation, NS2.

\section{Introduction}

A WSN (wireless sensor network) is a collection of mobile or static nodes that cooperate and communicate by wireless links deployed randomly or deterministically in a geographical area in order to detect, collect and transmit data about a phenomenon observed during a sink node (base station. The routing is one of the critical technologies in WSNs because: the resources are constrained in terms of energy, processing, storage capacities and transmission bandwidth, it's difficult to design a global addressing scheme as IP (internet protocol) for a large number of sensor nodes because the short addressing scheme is not scalable and the allocation process cannot guarantee the uniqueness of the address, as data collection by sensor nodes usually results in a high probability of data redundancy. In general, network structure routing in WSNs can be classified into three categories namely flat based routing, hierarchical based routing and location-based routing [1, 2].

In this research paper we have analyzed the performance of LEACH, LEACH-C and PEGASIS routing protocol and we have studied the effect of

Corresponding author : Hassan Echoukairi, student, research field : wireless sensor networks (wsn), distributed Systems. specific parameters such as energy dissipation, Throughput, Packet Delivery Ratio and End-to-End Delay on the performance of this three protocols. For this purpose we have simulated a WSN with various nodes density and with a base station, which coordinates first the center of the sensor field, the edge of the field and finally outside the field.

This paper is organized as follows: In Section 2, we describe the hierarchical routing protocols: LEACH, LEACH-C and PEGASIS; In Section 3, we define the different metrics of performances related to the WSN; The simulations results are discussed in Section 4; Finally, we offer a conclusion of the paper in Section 5.

\section{Hierarchical Routing Protocols in WSN}

\subsection{LEACH:}

In Ref. [3], LEACH (low-energy adaptive clustering hierarchy) is the first cluster-based routing protocol for WSN proposed by Heinzelman et al. in 2000. LEACH operations can be divided into two phases. The operation of LEACH is divided into rounds with each round having a setup phase where the clusters are formed and a $\mathrm{CH}$ (cluster-head) is chosen for each cluster and a steady state phase where data is sensed and sent to the central base station. Set-up Phase is 
divided into 2 parts as shown in Fig. 1

\section{Advertisement Phase}

Each node decides whether or not to become a $\mathrm{CH}$ for a round based on a threshold T(n).

- Each node $n$ generates a random number between 0 and 1 . If the random number is less than a threshold $\mathrm{T}(\mathrm{n})$ then the node elects itself to be a cluster head.

$$
T(n)=\left\{\begin{array}{c}
\frac{P}{1-P *\left(r \bmod \frac{1}{P}\right)}, \text { if } \mathrm{n} \in \mathrm{G} \\
0, \text { else }
\end{array}\right.
$$

$\mathrm{P}$ : desired percentage of cluster heads for round ( $\mathrm{P}$ $=0.05)$.

$\mathrm{r}$ : current round

$\mathrm{G}$ : is the set of nodes that have not been cluster headed in last $1 / \mathrm{P}$ rounds

- Each $\mathrm{CH}$ broadcasts advertisement message $(\mathrm{ADV}=$ node's ID + distinguishable header $)$ to rest of the nodes

- Non-CH node joins the cluster head with "Join_REQ" that contains its ID+ cluster-head ID + header and using CMSA which offers the high Received Signal Strength.

Cluster Set-up Phase:

- $\mathrm{CH}$ knows the number of member nodes and their IDs

- $\mathrm{CH}$ creates a TDMA schedule Based on all messages received and broadcasts the TDMA table to cluster members.

\section{In Steady Phase:}

- Non-CH nodes start sensing data and send it to their cluster-head during their allocated TDMA slot.

- When all the data has been received from member nodes, the $\mathrm{CH}$ aggregates it and sends it to the $\mathrm{BS}$ through a high energy transmission.

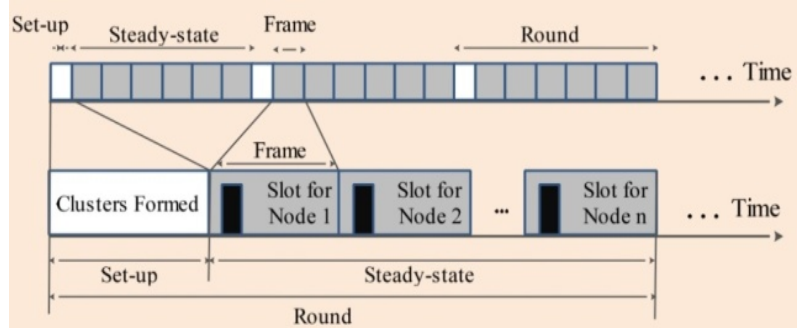

Fig. 1 Two phases operation in a round of LEACH.

\section{$2.2 \mathrm{LEACH}-\mathrm{C}$}

In Ref. [4], LEACH-C is centralized routing protocol which is based at centralized clustering algorithm for cluster formation. Its steady state phase is the same of LEACH protocol. In set up phase each node transmits its current position and residual energy level to the BS. The BS runs the centralized cluster formation algorithm to determine cluster heads and clusters for that round. Simulated annealing is used by LEACH-C to find close optimal clusters. LEACH-C determines cluster heads randomly, the BS makes sure that only nodes with more energy are participating in the cluster head selection. Once the clusters are created, the base station broadcasts the information to all the nodes in the network. Each of the nodes, except the cluster head, determines its TDMA slot used for data transmission. The node goes to sleep until it is time to transmit data to its cluster head.

\subsection{PEGASIS}

PEGASIS (power-efficient gathering in sensor information system) [5], are the typical protocols hierarchical-based routing proposed by Lindsey and Ragha-vendra in 2002. It is developed based on LEACH. It is a classical chain-based routing protocol that is an improvement over LEACH and it forms a chain among the sensor nodes. Each node communicates with a close neighbor and takes turns being the leader in transmission to the base station. This approach reduced the average energy spent by each node per round. The operation detail is done in two steps:

\section{Chain Construction :}

The chain construction starts from the furthest node from the BS by using the greedy approach to construct this chain.

The nearest neighbor to this node is the next node in the chain. All the nodes are included in the chain by the same manner and the chain is reconstructed bypass the dead node.

Gathering Data: 
In this step, each node receives data from one neighbor, fuses the data with its own, and transmits to the other neighbor on the chain. In each round, the leader node chosen randomly forwarded the aggregated data to the base station. Choosing leader node randomly also provides benefit as it is more likely for nodes to die at random locations; thus making a robust network for failures.

\section{Performances Metrics}

The performances of routing protocols [6] was evaluated on the basis of the performance metrics as is the case in Fig. 2:

Each of these metric parameters used in our simulation can be described briefly as follows:

\section{Average Energy Consumption:}

The average energy consumption is the average difference between the initial level of energy and the final level of energy that is left in each node. It's calculated by the following Eq. (2)

$$
E_{a}=\frac{1}{N} \sum_{k=1}^{N}\left(E_{i k}-E_{f k}\right)
$$

$\mathrm{Ei}=$ the initial energy level of a node, $\mathrm{Ef}=$ the final energy level of a node. $\mathrm{N}=$ number of nodes in the simulation. This metric is important because the energy level of the network used is proportional to the network's lifetime.

\section{Throughput:}

Throughput is the number of (average data) packet received at the base station per bit/sec and byte/sec generated by source. It's calculated by the following Eq. (3):

Throughput $($ Bit $/ \mathrm{s})=($ Number of Delivered Packet

* Packet size*8) / Total duration of simulation

PDR (packet delivery ratio):

It is the ratio between the number of packets that received by base station and the number of packets sent by the source. It is calculated by this formula:

Packet Delivery Rate (\%) = received packets number / sent packets number

\section{Simulations and Analysis}

This section describes the simulation tool, simulation parameters and simulation results. The performances of routing protocols was evaluated on the basis of three performance metrics: energy dissipation, throughput, PDR (packet delivery ratio).

Simulation Tool:

NS2 (network simulator version 2) [7-9], is a packet level discrete event simulator, developed by UC Berkeley is a widely used tool for computer simulations. It's becoming one of the first selected software to implement network simulation in the academic field. NS2 is a software package including some basic components like Tcl/Tk, OTcl, NS2, Tclcl, etc. As shown in the Fig. 3, the basic structure of NS-2 is composed of:

\section{Simulation Parameters:}

For our simulation, all nodes are homogeneous and randomly distributed on $100 \mathrm{~m} \times 100 \mathrm{~m}$ area with initial energy of 2 Joule. In each scenario, the BS (base station) is located at $(50,175) \mathrm{m},(50,75) \mathrm{m}$ and $(50,50) \mathrm{m}$.

The rest of following simulation parameters are used in this paper to analyze the performance of routing protocols as shown in the Table 1.

\section{Simulation Results and Discussion:}

The Results of the simulation for each location of base stations evaluate the performances metrics: average energy consumption, average throughput, PDR (\%) and

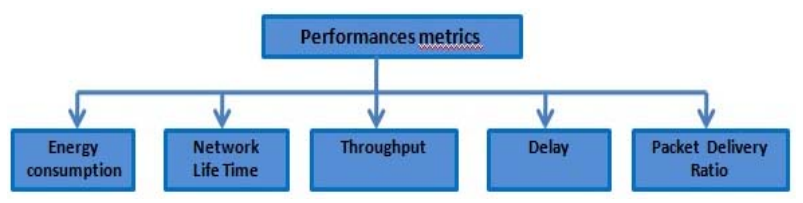

Fig. 2 Performances metrics.

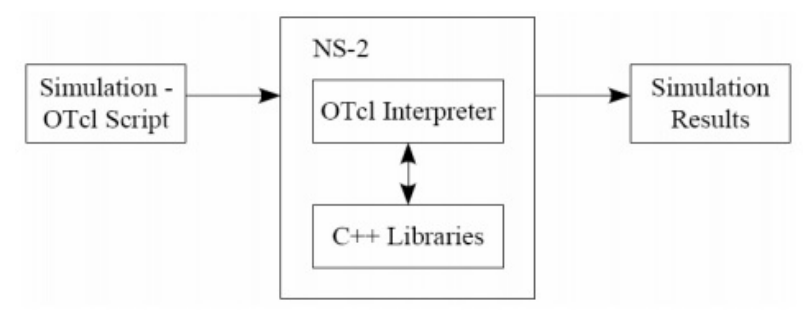

Fig. 3 Basic structure of NS-2. 
Table 1 Simulation parameters.

\begin{tabular}{|l|l|}
\hline PARAMETER & VALUE \\
\hline Number of nodes & $60,80,100,120,140$ \\
\hline Simulation time & $1000 \mathrm{~ms}$ \\
\hline Routing protocol & LEACH, LEACH-C, PEGASIS \\
\hline Simulation model & Two Ray Ground \\
\hline MAC Type & 802.11 \\
\hline Link Layer Type & LL \\
\hline Interface Type & Queue \\
\hline Packet Size & $512 \mathrm{MB}$ \\
\hline Queue Length & 50 packets \\
\hline
\end{tabular}

different density of nodes.

In these scenarios, we study the effect of varying the nodes density with different scenarios of location on the base station through the performance of LEACH, LEACH and PEGASIS.

Scenario A: Average Energy Consumption, Average Throughput and PDR versus Nodes Density for Position (50.175) of the Base Station

Scenario B: Average Energy Consumption, Average Throughput and PDR versus Nodes Density for Position (50.75) of the Base Station

Scenario C: Average Energy Consumption, Average Throughput and PDR versus Nodes Density for Position (50.50) of the Base Station

In order to compare the performances between LEACH, LEACH-C and PEGASIS in terms of average energy consumption, average throughput and PDR , we simulated them using NS2.34.

In the first scenario, we have observed in Fig. 1(a) that PEGASIS has almost constant consumption of the energy and more than both LEACH and LEACH-C routing protocols for different number of sensor nodes. When the number of nodes increases in the networks the energy consumption increases.

In PEGASIS, the throughput is very low as shown in Fig. 1(b) but also increases with increase in density like LEACH and LEACH-C protocols. The PDR in LEACH, LEACH-C and PEGASIS protocols decreases or increases with respect to the number of nodes as shown in Fig. 1(c).

In the second scenario, we have observed in Fig. 2(a) that it has got an almost constant consumption of energy that increases as the number of nodes increases. In Fig. 2(b), the throughput is almost constant and very lower with regard to other protocols. But in LEACH and LEACH-C, it increases gradually as the number of nodes increases. The PDR is lower in LEACH-C with regard to LEACH and PEGASIS routing protocols in Fig. 2(c).

In the third scenario, the energy consumption is constant and very higher in PEGASIS but it increases

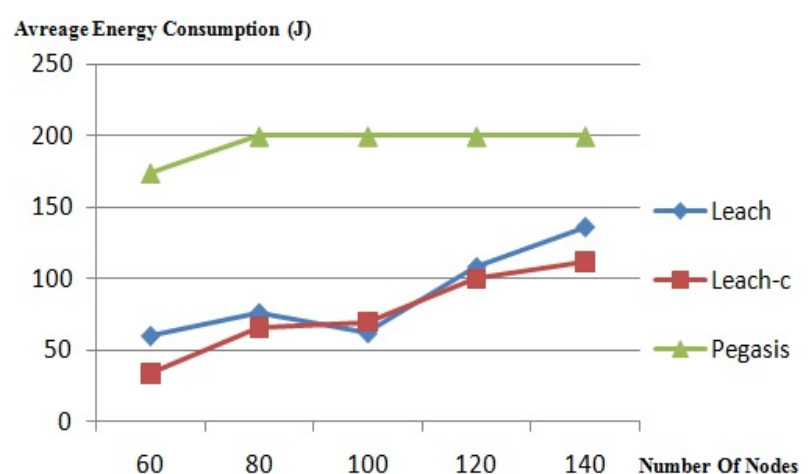

Fig. 1 (a) Average energy consumption vs. Number of nodes for BS $(50,175)$.

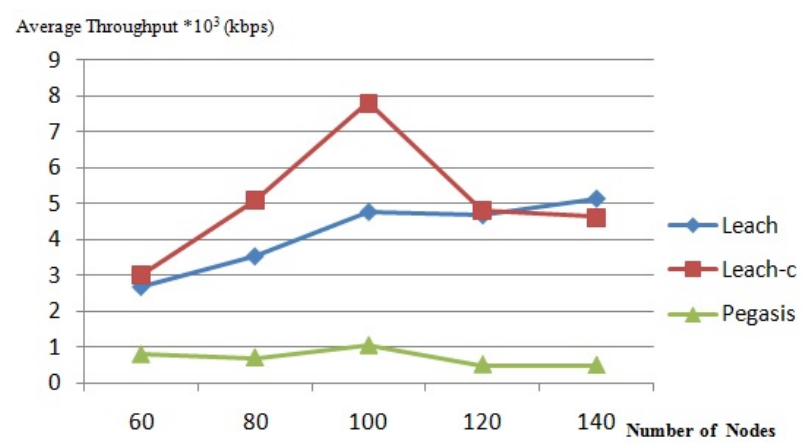

Fig. 1 (b) Average throughput vs. Number of nodes for BS $(50,175)$.

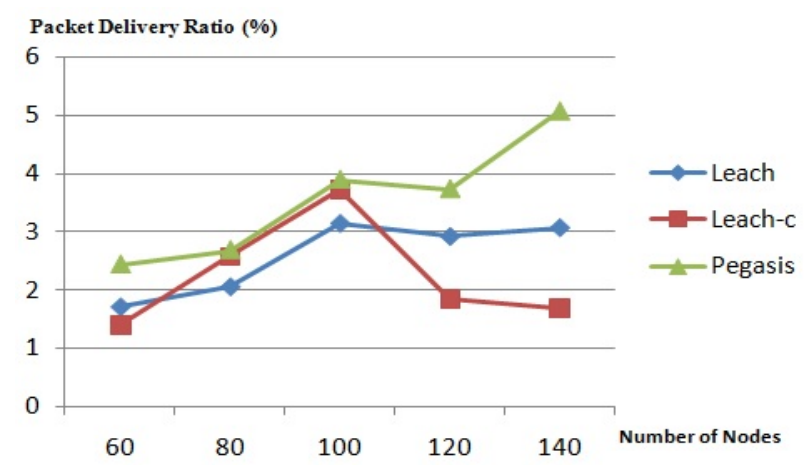

Fig. 1 (c) Packet delivery ratio vs. Number of Nodes or BS $(50,175)$. 


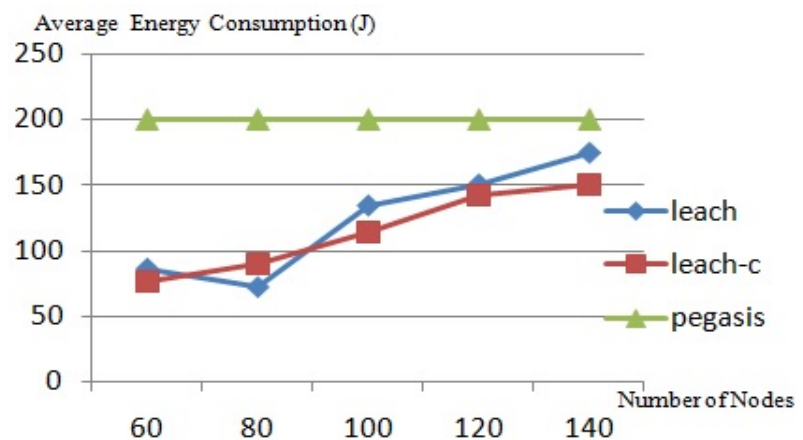

Fig. 2 (a) Average energy consumption vs. Number of nodes for BS $(50,75)$.

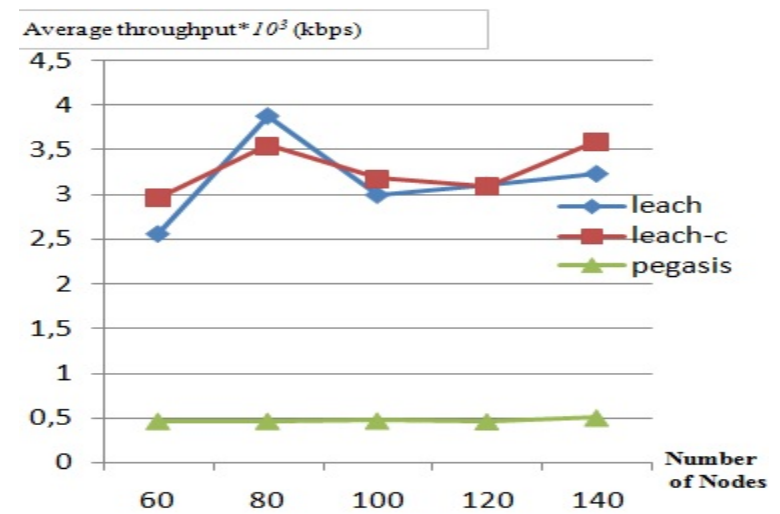

Fig. 2 (b) Average throughput vs. Number of nodes for BS $(50,75)$.

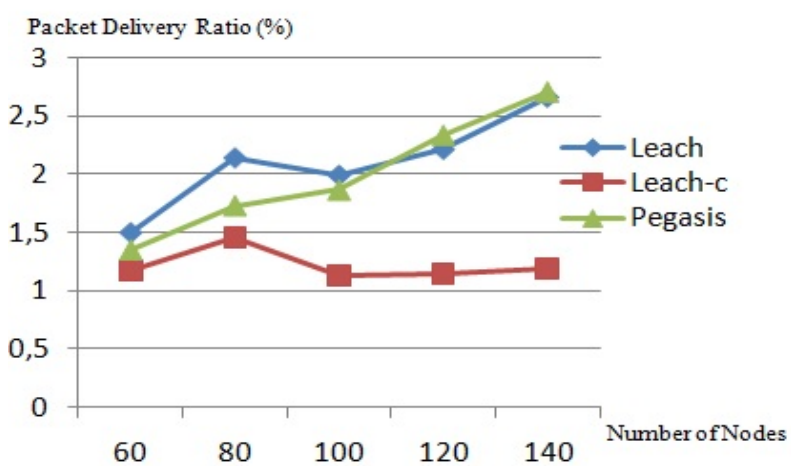

Fig. 2 (c) Packet delivery ratio vs. Number of nodes for BS $(50,75)$.

in LEACH and LECAH-C when the number of nodes increases as shown in Fig. 3(a).The throughput in Fig. 3(b) is lower in PEGASIS but increases with the increase in number of nodes like LECAH and LEACH-C protocols. The PDR in Fig. 3(c) is lower and almost constant in LECAH-C but increases in LEACH and PEGASIS when the number of nodes increases.

Thus the ideal value for average energy consumed by the protocol should be as less as possible, it indicates

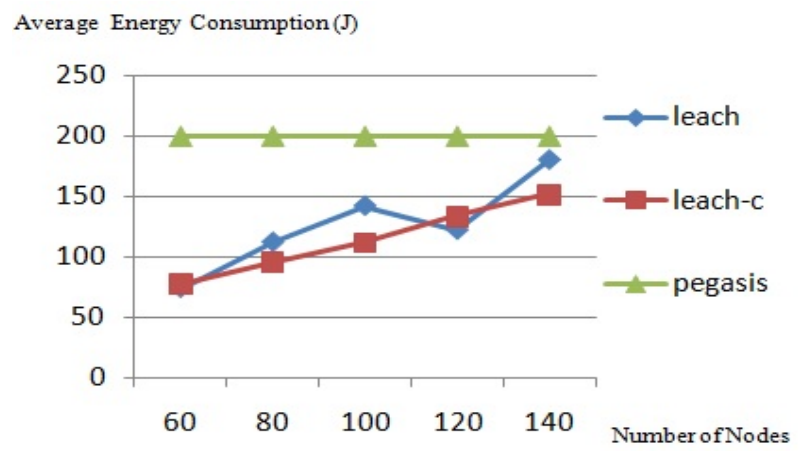

Fig. 3 (a) Average energy consumption vs. Number of nodes for BS $(\mathbf{5 0}, \mathbf{5 0})$.

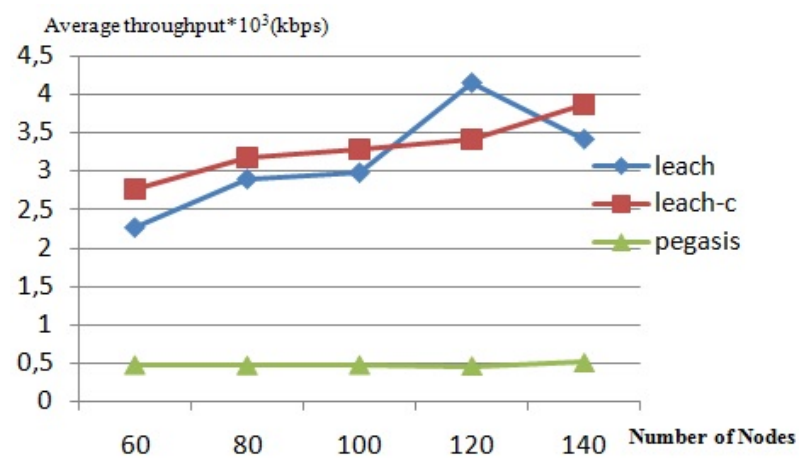

Fig. 3 (b) Average throughput vs. Number of nodes for BS $(50,50)$.

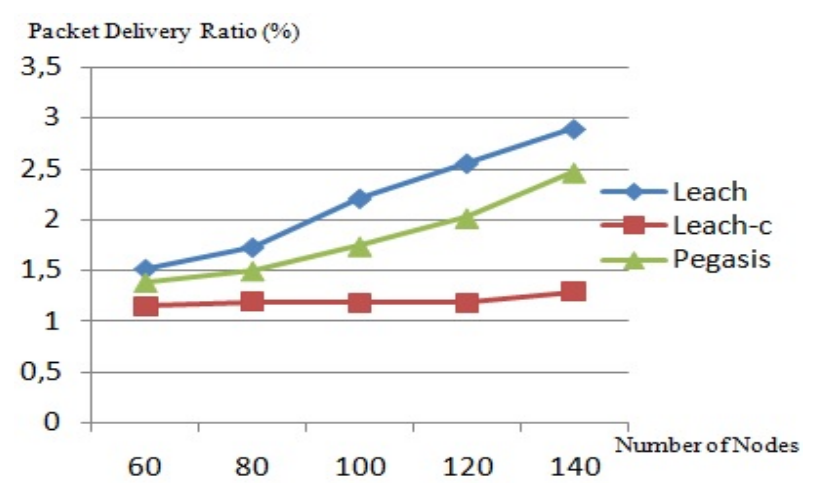

Fig. 3 (c) Packet delivery ratio vs. Number of nodes for BS $(50,50)$.

the network lifetime. In all these three scenarios, LEACH and LECAH-C protocols are better than PEGASIS in energy consumption.

The throughput defines the average of successfully delivering data packets in a network. In All three scenarios, LEACH and LECAH-C protocols are better than PEGASIS in this metric because they provide higher value.

When the PDR is higher, the performance of the networks is good. Outside the field, PEGASIS is 
better than LECAH and LECAH-C, but both at the edge and at the center of the field, PEGASIS and LECAH are better than LECAH-C.

Simulation results illustrate that the routing protocols varies across different node densities and base station locations, while LEACH-C and PEGASIS outperform LEACH with respect to a number of metrics.

\section{Conclusion}

In this work, simulation results provide an insight into varying the location of base stations and the number of nodes over the network and analyzing its impact on the various performance metrics. In all three scenarios, LEACH and LECAH-C protocols are better than PEGASIS in energy consumption and throughput. PEGASIS and LECAH protocols are better than LECAH-C in term of PDR.

For further perspective, we will try to evaluate the performance of wireless sensor routing protocols with different mobility patterns, different types of traffic and other metrics.

\section{References}

[1] Kaur, N. 2013. “Classification of Hierarchical Routing
Protocols in Wireless Sensor Network: A Survey.” International Journal of P2P Network Trends and Technology (IJPTT) 3 (1): 56-61.

[2] Davel, P., and Dalal, P. 2013. "Simulation \& Performance Evaluation of Routing Protocols in Wireless Sensor Network.” International Journal of Advanced Research in Computer and Communication Engineering 2 (3).

[3] Heinzelman, W. R., Chandrakasan, A., and Balakrishnan, H. 2000. "Energy Efficient Communication Protocol for Wireless Microsensor Networks.” In Proceedings 33rd Hawaii Inter-national Conference on System Sciences, 3005-14.

[4] Heinzelman, W. B., Chandrakasan, A. P., Member, S. and Balakrishnan, H. 2002. "An Application-Specific Protocol Architecture for Wireless Microsensor Networks." IEEE Transactions on Wireless Communications 1 (4): 660-70.

[5] Lindsey, S., Raghavendra, C., and Sivalingam, K. M. 2002. "Data Gathering Algorithms in Sensor Networks Using Energy Metrics.” IEEE Transactions on Parallel and Distributed Systems 13 (9): 924-35.

[6] Alwan, H., and Agarwal, A. 2013. "MQoSR: A Multiobjective QoS Routing Protocol for Wireless Sensor Networks.” ISRN Sensor Networks 2013: 12.

[7] Bin, Y., Bin, S., and Nuan, W. 2007. NS2 and Network Simulation. Beijing: Posts \& Telecom Press.

[8] Mark, G. 2003. "Marc Greis Tutorial for the UCB-LBNL-VINT Network Simulator.” at http://www.isi.edu/nsnam/ns/tutorial/index.html .

[9] The network simulator - ns-2. http:// www. isi.edu/nsnam/ns/. 